\title{
Food gum based hydrogel polymers
}

\begin{abstract}
Food gum, a kind of very important and unique food additive, has many important functions on food applications. In addition, they are also applied on non-food industries such as cosmetics, coating, photosensitive resin, fertilizer, casting, tobacco and pharmaceutical industries, due to their great structural versatility, high wateraffinity. Among these non-food applications, synthesis hydrogel polymers have been widely concerned due to their widespread applications in pharmaceutical and medical areas, e.g., as delivery vehicles for drugs, cells, genes, or proteins, and as scaffolds for tissue engineering. In this review, we summarized the food gum based hydrogel polymers such as gum ghatti based hydrogels, tragacanth gum based hydrogels, salecan gum based hydrogels, pectin based hydrogels, and guar gum based hydrogels. Based on the literatures reviewed, it is concluded that various food gum based hydrogels can be exploited as potential applications on medical areas, tissue engineering, and environment.
\end{abstract}

Keywords: food gum, hydrogel, application, gum ghatti
Volume 4 Issue 6 - 2017

\author{
Ai Jun Xie, Chun Yan Zhu, Hua Min Liu \\ College of Food Science and Technology, Henan University of \\ Technology, China
}

Correspondence: Hua Min Liu, College of Food Science and Technology, Henan University of Technology, Zhengzhou, China, Tel +86-037I-67789937, Fax ++86-037I-67789937, Email liuhuamin5108@163.com

Received: May 04, 2017 | Published: September 15, 2017
Abbreviations: Gg, gum ghatti; TG, tragacanth gum; PAAM, pure polyacrylamide; MWCNT, multiwall carbon nanotube

\section{Introduction}

Food gum, a kind of essential food additive, has many important functions on food applications, such as thickening, gelation, emulsification, suspension, stabilization and clarification, which can be widely used in frozen food, dairy products, beverages, condiments, cakes, starch, candy, wine, food and frozen food industry. ${ }^{1-5}$

Among these non-food applications, synthesis hydrogel polymers have been widely concerned due to their widespread applications in pharmaceutical and medical areas, e.g., as delivery vehicles for drugs, cells, genes, or proteins, and as scaffolds for tissue engineering. ${ }^{6-8}$ Hydrogels are polymeric materials that have a three-dimensional network structure with the ability of absorbing abundant water while maintain their integrity due to chemical and/or physical crosslinking. ${ }^{9-11}$ Polysaccharides such as various food gum have some favorable features, such as great structural versatility, high wateraffinity, and easy procedures, which facilitates the general applications of polysaccharide-based materials in the pharmaceutical field. ${ }^{12,13}$ Therefore, polysaccharide-based hydrogels have biodegradability, biocompatibility, high water content, and cellular integrations to afford a favorable microenvironment for cell differentiation and growth, which are quite promising in tissue engineering and biomedical application..$^{14,15}$

Several authors have studied and reviewed the synthesis and applications of various hydrogel polymers. But until now, there is no specific review regard to the food gum based hydrogel polymers, as well as their applications. In this review, we summarized the food gum based hydrogel polymers and their applications.

\section{Hydrogel synthesis methods}

To prepare successfully hydrogels with the desired physical and biological properties, several factors such as a biodegradability, biocompatibility, mechanical strength, sterilizability, water content (degree of swelling), drug loading capacity, and the components of the hydrogels, must be carefully considered..$^{16,17}$ The preparation methods of hydrogel polymers can be divided into physical and chemical crosslinking. The physically preparation hydrogel is formed by hydrogel bonds, while chemical crosslink method is cross-linked of high molecular chain by chemical bond linking under the conditions of light, heat, radiation and crosslink agents. The formation of gel by chemical crosslinking can be divided into two processes. One process is formed by crosslinking at the same time of polymerization, which prepared usually by the multifunctional monomer through the bulk polymerization or free radical polymerization. Another formation process of chemical crosslinking gel is formed linear polymer at first, and then followed by cross-linking between polymers to form a gel as shown in Table 1 . The hydrogel polymers obtained by two polysaccharides can be divided into interpenetrating polymeric networks and semi-interpenetrating polymeric networks. Interpenetrating polymeric network hydrogel is a kind of unique polymer blend, which is made up two cross-linked polymers by continuously interspersed with each other. For semi-interpenetrating polymeric network hydrogels, there is only one kind of crosslinked polymer network, and another is a linear and non crosslinked polymer. ${ }^{18}$ The crosslinking schematics of macromolecular polysaccharide-based hydrogels are shown in Figure 1.18

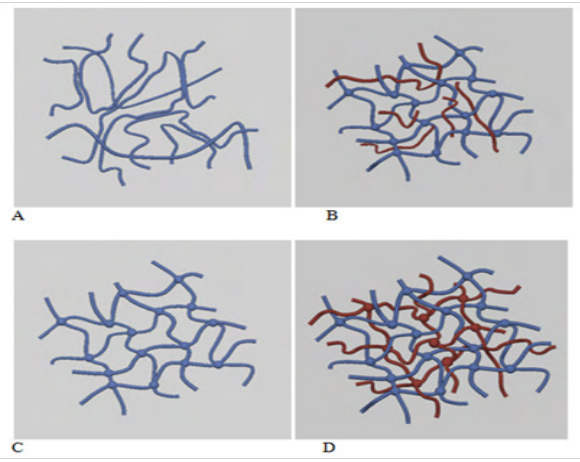

Figure I Crosslinking schematics of macromolecular polysaccharide-based hydrogels.

A) Physical crosslinking.

B) Chemical crosslinking.

C) Semi-interpenetrating polymeric networks.

D) Interpenetrating polymeric networks). ${ }^{18}$ 


\section{Food gum based hydrogels and their applications}

Gum ghatti based hydrogels: Gum ghatti $(\mathrm{Gg})$ is a complex anionic polysaccharide, which obtained from the exudation of Anogeissus latifolia tree (Combretaceae family). The primary structures of Gg have main chain of $1 \rightarrow 6$ linked $\beta$-D-galactopyranose units and alternating 4-O-subsituted and 2-O-subsituted a-D-mannopyranose units with the side chain of single L-arabinofuranose units. ${ }^{19}$ In food industry, it is mainly used as an additive, thickening agent and preservative. Recently, many investigations have been carried out on gum ghatti based hydrogels, such as Gum ghatti/poly(acrylamide-co-acrylic acid), gum ghatti/poly(acrylamide-co-acrylonitrile), gum ghatti-clpoly(acrylamide), ghatti-cl-Poly (acrylamide -co-methacrylic acid, and gum ghatti crosslinked with poly(acrylic acid-co-acrylamide), and the resulting hydrogel polymers have potential applications in the drug delivery devices, and waste water treatment. ${ }^{20-22}$ In addition, the synthetic mechanism of these hydrogels is also described in the published papers. For gum ghatti/poly (acrylamide-co-acrylic acid), the synthetic mechanism of the hydrogel is shown in Figure $2 .{ }^{19}$ As shown, ascorbic acid and KPS were used as a redox initiator and MBA was used as a crosslinking agent in the graft co-polymerization method. Initially, the ascorbic acid ions reacted with KPS and generate $\mathrm{SO}_{4}^{-*}$, which upon subsequent reactions with water molecules, generated $\mathrm{OH}^{*}$. Afterwards, the interaction of $\mathrm{OH}^{*}$ and $\mathrm{SO}_{4}^{-*}$ with $\mathrm{Gg}$ and monomers generated active sites for graft co-polymerization. Activated P (AAM-co-AA) and Gg free radicals would further react and form a crosslinked hydrogel polymer. However, chain-termination reactions might occur, either through the reaction of $\mathrm{OH}^{*}$ with live propagating macromolecular chains or through reactions between two activated chains. $^{19}$

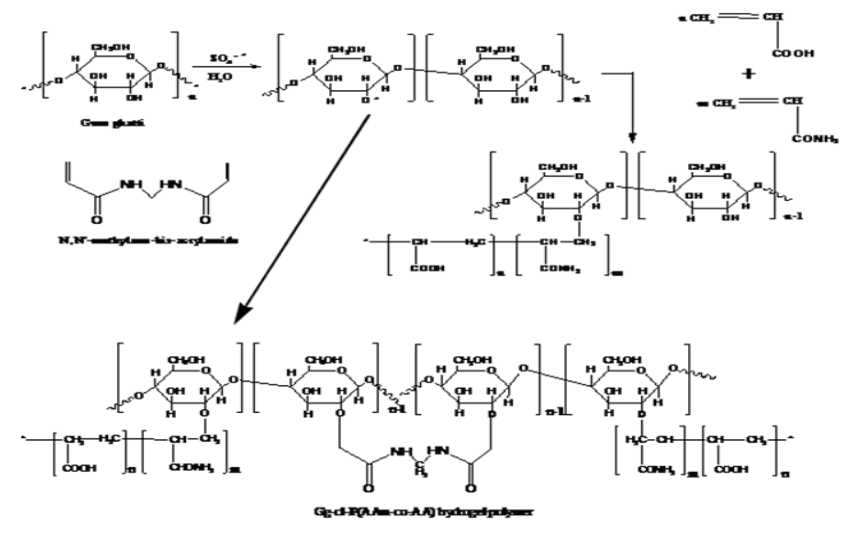

Figure 2 Mechanism for the synthesis of Gg-cl-P (AAM-co-AA). ${ }^{19}$

Tragacanth gum based hydrogels: Tragacanth gum (TG) is nonmutagenic, non-allergenic, non-carcinogenic, non-teratogenic and non-toxic natural hydrophilic polysaccharide and it makes a suitable medium for cell growth. ${ }^{23}$ It is a highly branched, heterogeneous, complex polysaccharides and comprising of galacturonic acid, xylose, arabinose, galactose and fucose. The primary and secondary hydroxyl and carboxylic acid groups present in tragacanth gum TG molecules provide positions for reacting with monomers and crosslinking reagents. ${ }^{24,25}$ Singh et al. ${ }^{26}$ for example, reported the preparation of tragacanth gum based sterile hydrogel by radiation method and the prepared hydrogel polymer could be used in drug delivery and wound dressing. The results showed that the composition of the hydrogel polymer influenced the hydrogel swelling. These prepared hydrogels wound dressings absorbed simulated wound fluid and were found to be blood compatible, non-thrombogenic nature. In addition, these hydrogels entrapped and released the model drug moxi-floxacin in a controlled manner for $24 \mathrm{~h}$ period without any sudden release. Therefore, these hydrogels can be considered as an appropriate material for wound dressing application, due to its controlled drug release, high swelling debridement properties and its ability to maintain optimal wound healing environment to step up the wound healing process.

Salecan gum based hydrogels: Salecan is a linear $(1 \rightarrow 3)-\beta$-D-glucan comprising $\beta-1-3$-linked glucopyranosyls with a small number of $\alpha-1$ 3 -linked. ${ }^{27}$ As novel microbial polysaccharides, Salecan has excellent biological activities including antoxidation and non-toxicity (edible safety). Salecan has unique physic-chemical properties, which can be utilized in both the food and medical fields. The solution of salecan has high viscosity and its molecular weight is $2 \times 10^{6} \mathrm{Da}$. In addition, Salecan contains a high density of hydroxyl groups, which can be modified to provide greater flexibility in the preparation of hydrogels. More importantly, the high solubility of Salecan allows large amounts of functional groups to be incorporated onto the polysaccharide chains without compromising its solubility in water. These features make Salecan a promising candidate for fabricating hydrogels used in different biotechnological applications ${ }^{28} \mathrm{Hu}$ et al. ${ }^{28}$ observed that cells could not grow on the surface of the pure polyacrylamide (PAAm) hydrogel, but it could adhere well on the surface of the salecan/PAAm semi-IPN hydrogels owing to the addition of salecan (Figure 3 ). ${ }^{28}$ Wei et al. ${ }^{29}$ reported a novel $\mathrm{pH}$-thermo dual responsive hydrogel based on salecan and poly (N,N-diethylacrylamide-co-methacrylic acid) semi-interpenetrating polymer networks (semi-IPNs). The results showed that it was non-toxic while the drug released from hydrogels had comparable cytotoxicity with respect to free drug. Therefore, the novel salecan/poly (N,N-diethylacrylamide-co-methacrylic acid) semi-interpenetrating polymer network hydrogels were $\mathrm{pH}$-thermo dual responsive and may be a promising candidate for drug delivery system.

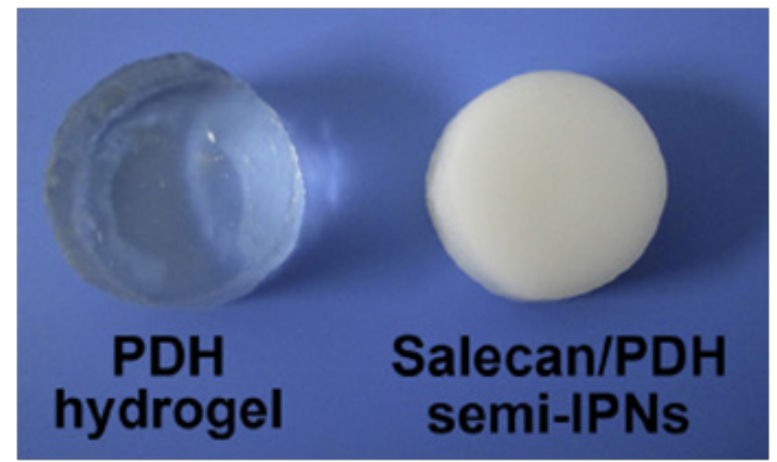

Figure 3 Photograph images of pure polyacrylamide and salecan/PAAm semiIPN hydrogels. 28

Pectin based hydrogels: Pectin is an anionic polysaccharide hydrogel, poly (1,4-galacturonic acid), found in the cell walls of terrestrial plants, which has been traditionally used in food industry. ${ }^{30}$ Recently, it has also become a material of interest from the biomedical point of view. Tripathi et al..$^{31}$ fabricated chitosan/poly (vinyl alcohol)/pectin ternary films and showed that the films have excellent antimicrobial properties for food packaging applications. Hydrogel microspheres may be useful for a variety of applications within the personal care, food, and pharmaceutical industries. ${ }^{32,33}$ In addition, they may also be used to protect, encapsulate, and release bioactive agents, such as 
pharmaceuticals, cosmetics, or nutraceuticals. Wu et al. ${ }^{34}$ demonstrated the potential of using hydrogel microspheres formed from gelatin and pectin to replace fat and starch granules. These hydrogel particles can be formed via electrostatic complexation (Figure 4). ${ }^{34,35}$ Solution conditions are manipulated so that the gelatin and pectin molecules associate with each other through electrostatic attraction, which leads to phase separation into a polymer-rich phase and a polymer-depleted phase. These hydrogel particles may be promising candidates for the development of healthy reduced-calorie foods because they are assembled from proteins and dietary fibers.

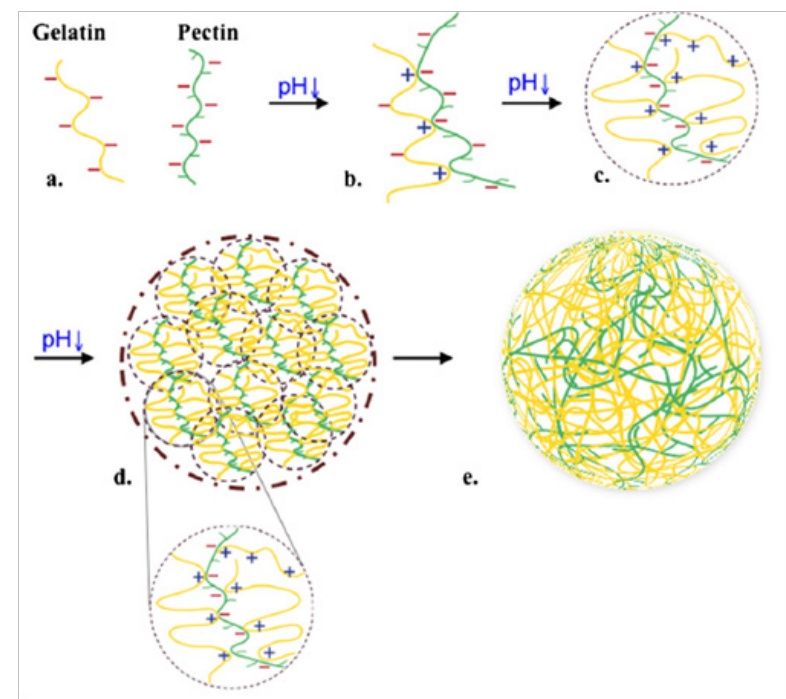

Figure 4 Schematic illustration of the electrostatic complexation that occurs during acidification of gelatin and pectin mixtures.

A. Gelatin and pectin molecules exist as individual molecules in solution due to electrostatic repulsion.

B. gelatin-pectin soluble complexes are formed due to electrostatic attraction between positive patches on gelatin and negative patches on pectin.

C. Soluble complexes merge and form gelatin-pectin sub-units.

D. Hydrogel particles form due to coalescence of sub-units.

E. Setting of internal structure as temperature cools down. ${ }^{34,35}$

Guar gum based hydrogels: Guar gum is a natural hydrophilic polysaccharide obtained from the endosperm of the guar plant, which offers an interesting set of characteristics such as non-toxicity, biodegradability, biocompatibility and bioactivity for different applications. ${ }^{36}$ Guar gum consists of linear chains of $(1 \rightarrow 4)-\beta-D-$ mannopyranosyl units with $\alpha$-D-galactopyranosyl units attached by $(1 \rightarrow 6)$ linkages (See Figure 5$) \cdot{ }^{37}$ Studies on guar gum-g-poly acrylamide microgels, modified by saponification, as $\mathrm{pH}$-responsive drug delivery system have been reported..$^{38}$ Oral controlled delivery systems for antihypertensive drug trimetazidine dihydrochloride using GG as a carrier were also reported. ${ }^{39}$ The results clearly indicate that GG in the form of a three layer matrix system is a potential hydrophilic carrier in the design of oral controlled drug delivery systems for highly soluble drugs. As a natural biopolymer, guar gum (GG) was also covalently grafted on the surfaces of multiwall carbon nanotube (MWCNT) to obtain GG-MWCNT composite. Then iron oxide nanoparticles were synthesized on the GG-MWCNT to prepare the magnetic GG-MWCNT-Fe ${ }_{3} \mathrm{O}_{4}$. The prepared GG-MWCNT$\mathrm{Fe}_{3} \mathrm{O}_{4}$ exhibited super paramagnetic with a saturation magnetization $(13.3 \mathrm{emu} / \mathrm{g})$, and good adsorption on neutral red and methylene blue, which could be easily separated from the aqueous solution in a magnetic field. ${ }^{39}$

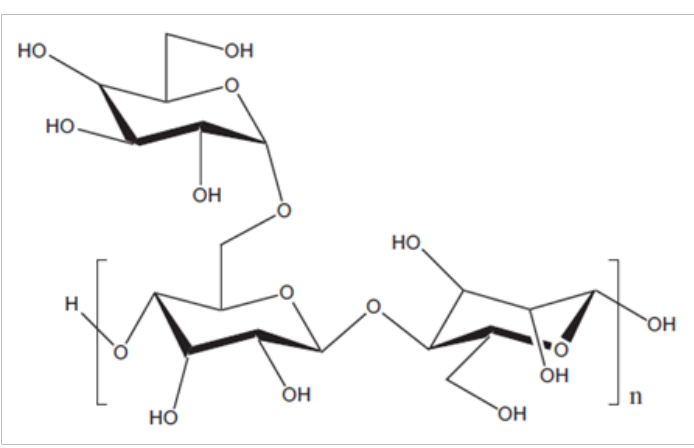

Figure 5 Structure of guar gum.

Table 1 Different methods of crosslinking polymers

\begin{tabular}{|c|c|c|}
\hline $\begin{array}{l}\text { Straight chain } \\
\text { polymer }\end{array}$ & $\begin{array}{l}\text { Reactive } \\
\text { functional group }\end{array}$ & Applicable cross linker \\
\hline $\begin{array}{l}\text { Polyacrylic acid, } \\
\text { Polyglutamic acid, } \\
\text { Polymethylacrylic } \\
\text { acid }\end{array}$ & $-\mathrm{COOH}$ & $\begin{array}{l}\text { Polyethylene glycol glycerol, } \\
\text { ethylene diamine }\end{array}$ \\
\hline $\begin{array}{l}\text { Poval, } \\
\text { 2-hydroxyethyl } \\
\text { polymethacrylate, } \\
\text { Hyaluronic acid, } \\
\text { Starch }\end{array}$ & $-\mathrm{OH}$ & $\begin{array}{l}\text { Glutaraldehyde, glutaric } \\
\text { acid, dimethylolurea, } \\
\text { ethylene glycol, bis } \\
\text { (2,3-epoxypropyl) ether }\end{array}$ \\
\hline $\begin{array}{l}\text { Allylamine } \\
\text { homopolymer, } \\
\text { Polylysine, } \\
\text { Collagen }\end{array}$ & $-\mathrm{NH}_{2}, \mathrm{NHR}$ & $\begin{array}{l}\text { ethylene glycol, bis } \\
\text { (2,3-epoxypropyl) ether, } \\
\text { dibromoethane, diiodoethane }\end{array}$ \\
\hline $\begin{array}{l}\text { Polyvinylpyridine, } \\
\text { Polyvinyl imidazol }\end{array}$ & -NRR' & $\begin{array}{l}\text { Ethylene glycol, } \\
\text { dibromoethane, bis } \\
\text { (2,3-epoxypropyl) ether }\end{array}$ \\
\hline
\end{tabular}

\section{Conclusion}

Food gum has some favorable features, such as great structural versatility, high water-affinity, and easy procedures, which facilitates the general applications of polysaccharide-based materials in the pharmaceutical field. Food gum-based hydrogels have biodegradability, biocompatibility, high water content, and cellular integrations to afford a favorable microenvironment for cell differentiation and growth, which are quite promising in tissue engineering and biomedical application. The review focused on food gum based hydrogel polymers such as gum ghatti based hydrogels, tragacanth gum based hydrogels, salecan gum based hydrogels, pectin based hydrogels, and guar gum based hydrogels. Based on the literatures reviewed, it is concluded that various food gum based hydrogels can be exploited as potential applications on medical areas, tissue engineering, and environment.

\section{Acknowledgements}

The authors gratefully acknowledge the support provided by Wang L, Qin Z of Zhengzhou University.

\section{Conflicts of interest}

The author declares no conflict of interest. 


\section{References}

1. Dickinson E. Hydrocolloids at interfaces and the influence on the properties of dispersed systems. Food Hydrocolloids. 2003;17(1):25-39.

2. Funami T. Next target for food hydrocolloid studies: texture design of foods using hydrocolloid technology. Food Hydrocolloids. 2011;25(8):1904 1914.

3. Nishinari K, Takahashi R. Recent advances in the understanding of heat set gelling polysaccharides. Trends in Food Science. 2004;15(6):305-312.

4. Wolever TMS, Tosh SM, Gibbs AL, et al. Physicochemical properties of oat $\beta$-glucan influence its ability to reduce serum LDL cholesterol in humans: a randomized clinical trial. Am J Clin Nutr. 2010;92(4):723-732.

5. Sawai R, Kuroda K, Shibata T, et al. Anti-influenza virus activity of Chaenomeles sinensis. J Ethnopharmacol. 2008;118(1):108-112.

6. Wei W, Qi XL, Liu YC, et al, Synthesis and characterization of a novel $\mathrm{pH}$-thermo dual responsive hydrogel based on salecan and poly(N,Ndiethylacrylamide-co-methacrylic acid). Colloids and Surfaces B: Biointerfaces. 2015;136:1182-1192.

7. Hemant M, Arjun M, Suprakas SR. Gum ghatti and poly (acrylamide-coacrylic acid) based biodegradable hydrogel-evaluation of the flocculation and adsorption properties. Polymer Degradation and Stability. 2015; 120:42-52.

8. Paulo AG, Soares JRP, Priscilla BSA, et al. Development and characterization of a new hydrogel based on galactomannan and $\mathrm{k}-$ carrageenan. Carbohydrate Polymers. 2015;134:673-679.

9. Zhou HY, Jiang LJ, Cao PP, et al. Glycerophosphate-based chitosan thermosensitive hydrogels and their biomedical applications. Carbohydr Polym. 2015;117:524-536.

10. Wang $\mathrm{B}, \mathrm{Xu} \mathrm{XD}$, Wang $\mathrm{ZC}$, et al. Synthesis andproperties of $\mathrm{pH}$ and temperature sensitive P(NIPAAm-co-DMAEMA)hydrogels. Colloids Surf. B Biointerfaces. 2008;64(1):34-41.

11. Chen R, Chen Q, Huo D, et al. In situ formation of chitosan-gold hybrid hydrogel and its application for drug delivery. Colloids Surf B Biointerfaces. 2012;97:132-137.

12. Yu YB, Pan H, Wang YY, et al. New insights into an innovative Auricularia auricular polysaccharide $\mathrm{pH}$-sensitive hydrogel for controlled protein drug delivery. RSC Adv. 2016;6(64):59794-59799.

13. Tao YZ, Zhang RQ, Yang W, et al. Carboxy methylated hyper branched polysaccharide: Synthesis, solution properties, and fabrication of hydrogel. Carbohydr Polym. 2015;128:179-187.

14. Diolosà $\mathrm{M}$, Donati I, Turco G, et al. Use of methacrylated-modified chitosan to increase the durability of dentinebonding systems. Biomacromolecules. 2014;15(12):4606-4613.

15. Zhang ZP, Zhang RJ, Zou LQ, et al. Encapsulation of curcumin in polysaccharide-based hydrogel beads: Impact of bead type on lipid digestion and curcumin bioaccessibility. Food Hydrocolloids. 2016;58:160-170.

16. Rezanejade Bardajee G, Pourjavadi A, et al. Novel highly swelling nanoporous hydrogel based on polysaccharide/protein hybrid backbone. Journal of Polymer Research. 2011;18(3):337-346.

17. Mohammad RS, Ghasem F, Saleh B, et al. Non-isothermal dehydration kinetic study of a new swollen biopolymer silver nanocomposite hydrogel. Journal of Thermal Analysis and Calorimetry. 2015;121(3):1383-1391.

18. Jiang JX, Liu YT, Zhou ZY, et al. Research progress and applications of macromolecular polysaccharidebased hydrogels for functional materials. Chemistry and Industry of Forest Products. 2017;37(2):1-10.
19. Mittal H, Shivani BM, Mishra AK, et al. Flocculation characteristics and biodegradation studies of Gum ghatti based hydrogels. International Journal of Biological Macromolecules. 2013;58:37-46.

20. Hemant M, Rajeev J, Balbir SK, et al. Synthesis and flocculation properties of gum ghatti andpoly(acrylamide-co-acrylonitrile) based biodegradable hydrogels. Carbohydr Polym. 2014;114:321-329.

21. Hemant M, Arjun M, Suprakas SR. Effective removal of cationic dyes from aqueous solution using gumghatti-based biodegradable hydrogel. International Journal of Biological Macromolecules. 2015;79:8-20.

22. Mittal H, Mishra SB. Gum ghatti and Fe3O4magnetic nanoparticles based nanocompositesfor the effective adsorption of rhodamine B. Carbohydrate Polymers. 2014;101:1255-1264.

23. Baljit S, Lalit V, Sanju F, et al. Designing tragacanth gum based sterile hydrogel by radiation method for use in drug delivery and wound dressing applications. International Journal of Biological Macromolecules. 2016;88:586-602.

24. Kiani A, Shahbazi M, Asempour H. Hydrogel membranes based on gum tragacanth with tunable structure and properties. I. Preparation method using Taguchi experimental design. Journal of Applied Polymer Science. 2012;124(1):99-108.

25. Großl M, Harrison S, Kaml I, et al. Characterisation of natural polysaccharides (plant gums) used as binding media for artistic and historic works by capillary zone electrophoresis. Journal of Chromatography A. 2005;1077(1):80-89.

26. Baljit S, Lalit V, Sanju F, et al. Designing tragacanth gum based sterile hydrogel by radiation method for use in drug delivery and wound dressing applications. International Journal of Biological Macromolecules. 2016;88:586-602.

27. Xiu AH, Kong Y, Zhou MY, et al. The chem-ical and digestive properties of a soluble glucan from A grobacterium sp.ZX09. Carbohydrate Polymers. 2010;82(3):623-628.

28. Hu XY, Feng LD, Wei W, et al. Synthesis and characterization of a novel semi-IPN hydrogel based onSalecan and poly(N,N-dimethylacrylamideco-2-hydroxyethylmethacrylate). Carbohydrate Polymers. 2014;105:135144.

29. Wei W, Qi XL, Liu YC, et al. Synthesis and characterization of a novel $\mathrm{pH}$-thermo dual responsive hydrogel based on salecan and poly $(\mathrm{N}, \mathrm{N}-$ diethylacrylamide-co-methacrylic acid). Colloids and Surfaces B: Biointerfaces. 2015;136:1182-1192.

30. You YC, Dong LY, Dong K, et al. In vitro and in vivo application of $\mathrm{pH}-$ sensitive colon-targeting polysaccharide hydrogel used for ulcerative colitis therapy. Carbohydr Polym. 2015;130:243-253.

31. Tripathi S, Mehrotra GK, Dutta PK. Preparation and physico-chemical evaluation of chitosan/poly (vinyl alcohol) / pectinternary film for foodpackaging applications. Carbohydrate Polymers. 2010;79(3):711-716.

32. Matalanis A, Mcclements DJ. Hydrogel microspheres for encapsulation of lipophilic components: Optimization of fabrication \& performance. Food Hydrocolloids. 2013;31(1):15-25.

33. Vashist A, Vashist A, Gupta YK, et al. Recent advances in hydrogel based drug delivery systems for the human body. Journal of Materials Chemistry B. 2014;2(2):147-166.

34. Wu BC, Degner B, McClements DJ. Soft matter strategies for controlling food texture: Formation of hydrogel particles by biopolymer complex coacervation. J Phys Condens Matter. 2014;26(46):464104.

35. Wu BC, David JM. Functional hydrogel microspheres: Parameters affecting electrostatic assembly of biopolymer particles fabricated from gelatin and pectin. Food Research International. 2015;72:231-240. 
36. Li Y, Chang PR, Zheng PW, et al. Characterization of magnetic guar gumgrafted carbon nanotubes and the adsorption of the dyes. Carbohydrate Polymers. 2012;87(3):1919-1924.

37. Goldstein AM, Alter EN, Seaman JK. In: RL Whistler, et al. editors. Industrial Gums, Polysaccharides and Their Derivatives. New York, USA: Academic Press; 1973. p. 303-321.
38. Soppiamath KS, Kulakarni AR, Aminabhavi TMA. Chemically modified polyacrylamide-g-guar gum-based crosslinked anionic microgels as $\mathrm{pH}$-sensitive drug delivery systems: preparation and characterization. $J$ Control Release. 2000;75(3):331-345.

39. Krishnaiah YSR, Karthikeyan RS, Sankar VG, et al. Three-layer guar gum matrix tablet formulations for oral controlled delivery of highly soluble trimetazidine dihydrochloride. J Control Release. 2002;81(1-2):45-56. 